\title{
Special Cases of the Parking Functions Conjecture and Upper-Triangular Matrices
}

\author{
Paul Levande $\|^{t}$ \\ ${ }^{1}$ Department of Mathematics, University of Pennsylvania, Philadelphia, PA, USA
}

\begin{abstract}
We examine the $q=1$ and $t=0$ special cases of the parking functions conjecture. The parking functions conjecture states that the Hilbert series for the space of diagonal harmonics is equal to the bivariate generating function of area and dinv over the set of parking functions. Haglund recently proved that the Hilbert series for the space of diagonal harmonics is equal to a bivariate generating function over the set of Tesler matrices-upper-triangular matrices with every hook sum equal to one. We give a combinatorial interpretation of the Haglund generating function at $q=1$ and prove the corresponding case of the parking functions conjecture (first proven by Garsia and Haiman). We also discuss a possible proof of the $t=0$ case consistent with this combinatorial interpretation. We conclude by briefy discusing possible refinements of the parking functions conjecture arising from this research and point of view. Note added in proof: We have since found such a proof of the $t=0$ case and conjectured more detailed refinements. This research will most likely be presented in full in a forthcoming article.

Résumé. On examine les cas spéciaux $q=1$ et $t=0$ de la conjecture des fonctions de stationnement. Cette conjecture déclare que la série de Hilbert pour l'espace des harmoniques diagonaux est égale à la fonction génératrice bivariée (paramètres area et dinv) sur l'ensemble des fonctions de stationnement. Haglund a prouvé récemment que la série de Hilbert pour l'espace des harmoniques diagonaux est égale à une fonction génératrice bivariée sur l'ensemble des matrices de Tesler triangulaires supérieures dont la somme de chaque équerre vaut un. On donne une interprétation combinatoire de la fonction génératrice de Haglund pour $q=1$ et on prouve le cas correspondant de la conjecture dans le cas des fonctions de stationnement (prouvé d'abord par Garsia et Haiman). On discute aussi d'une preuve possible du cas $t=0$, cohérente avec cette interprétation combinatoire. On conclut en discutant brièvement les raffinements possibles de la conjecture des fonctions de stationnement de ce point de vue. Note ajoutée sur épreuve: j'ai trouvé depuis cet article une preuve du cas $t=0$ et conjecturé des raffinements possibles. Ces résultats seront probablement présentés dans un article ultérieur.
\end{abstract}

Keywords: parking function, Hilbert series, diagonal harmonics

\section{Introduction}

A parking function of length $n$ is a sequence $a_{1} a_{2} \cdots a_{n}$ such that, for all $1 \leq i \leq n$,

$$
\left|f^{-1}(\{1,2, \cdots, i\})\right| \geq i \text {. }
$$

\footnotetext{
${ }^{\dagger}$ This research has been supported by the University of Pennsylvania Ph.D. program in Mathematics. 
Let $P_{n}$ be the set of parking functions of length $n$. The parking functions conjecture (see [Hag08]) states that the Hilbert series for the space of diagonal harmonics is given by the following bivariate generating function over $P_{n}$.

$$
\sum_{a_{1} a_{2} \cdots a_{n} \in P_{n}} q^{\operatorname{dinv}\left(a_{1} a_{2} \cdots a_{n}\right)} t^{\operatorname{area}\left(a_{1} a_{2} \cdots a_{n}\right)}
$$

where area and dinv are statistics defined on parking functions.

Let the $m$-th hook sum of an upper-triangular matrix $A=\left[a_{i, j}\right]_{1 \leq i \leq j \leq n}$ be given by

$$
\sum_{r=m}^{n} a_{m, r}-\sum_{k=1}^{m-1} a_{k, m} .
$$

Let $A$ be a Tesler matrix if and only if it has non-negative integer entries and every hook sum is equal to one. Let $T_{n}$ be the set of $n \times n$ Tesler matrices. The following matrix is an example of a member of $T_{5}$ :

$$
\begin{array}{lllll}
0 & 1 & 0 & 0 & 0 \\
& 0 & 1 & 0 & 1 \\
& & 1 & 1 & 0 \\
& & & 2 & 0 \\
& & & & 2 .
\end{array}
$$

Let $[n]_{q, t}$ be the polynomial $\sum_{i=0}^{n-1} q^{i} t^{n-1-i}$. If $A \in T_{n}$, let $\operatorname{extra}(A)$ be $n$ less than the number of nonzero entries of $A$. (By construction, $A$ must have at least one nonzero entry per row.)

Haglund $[\mathrm{Hag}]$ recently proved that the Hilbert series of diagonal harmonics is given by the following bivariate generating function over $T_{n}$ :

$$
\sum_{A=\left[a_{i, j}\right] \in T_{n}}((q-1)(1-t))^{\operatorname{extra}(A)} \prod_{a_{i, j}>0}\left[a_{i, j}\right]_{q, t} .
$$

As a result of Haglund's proof, the parking functions conjecture can now be considered equivalent to the following identity of bivariate generating functions:

$$
\sum_{a_{1} a_{2} \cdots a_{n} \in P_{n}} q^{\operatorname{dinv}\left(a_{1} a_{2} \cdots a_{n}\right)} t^{\operatorname{area}\left(a_{1} a_{2} \cdots a_{n}\right)}=\sum_{A=\left[a_{i, j}\right] \in T_{n}}((q-1)(1-t))^{\operatorname{extra}(A)} \prod_{a_{i, j}>0}\left[a_{i, j}\right]_{q, t} .
$$

In the following extended abstract, we will prove the $q=1$ special case of Equation (1) and give an outline of a proof of the $t=0$ special case. The $q=1$ special case was first proven by Garsia and Haiman [GH96.

\section{The $q=1$ special case}

Let $T_{n}^{*}=\left\{A \in T_{n}: \operatorname{extra}(A)=0\right\}$. Alternately, let $T_{n}^{*}$ be the set of $n \times n$ Tesler matrices with precisely one nonzero entry per row. Let $[n]_{t}=\left.[n]_{q, t}\right|_{q=1}=\sum_{i=0}^{n-1} t^{i}$. For $a_{1} a_{2} \cdots a_{n} \in P_{n}$, let area $\left(a_{1} a_{2} \cdots a_{n}\right)=\left(\begin{array}{c}n+1 \\ 2\end{array}\right)-\sum_{i=1}^{n} a_{i}$.

When Equation (1) is evaluated at $q=1$, the following equation results: 


$$
\sum_{a_{1} a_{2} \cdots a_{n} \in P_{n}} t^{\text {area }\left(a_{1} a_{2} \cdots a_{n}\right)}=\sum_{A=\left[a_{i, j}\right] \in T_{n}^{*}} \prod_{a_{i, j}>0}\left[a_{i, j}\right]_{t} .
$$

To prove Equation (2), we will begin by constructing a surjective map $\phi$ from $P_{n}$ to $S_{n}$. (Note added in proof: Haglund pointed out that this map sends each parking function to the inverse of the permutation it "parks" to. We will retain our original description.) We will then give a simple proof that $\left|T_{n}^{*}\right|=n !$. We will then construct a bijection between $S_{n}$ and $T_{n}^{*}$ by associating each permutation $\pi \in S_{n}$ to a unique Tesler matrix $C_{\pi} \in T_{n}^{*}$. Finally, we will prove that, for each $\pi \in S_{n}$,

$$
\sum_{\phi\left(a_{1} a_{2} \cdots a_{n}\right)=\pi} t^{\text {area }\left(a_{1} a_{2} \cdots a_{n}\right)}=\prod_{C_{\pi}=\left[a_{i, j}\right], a_{i, j}>0}\left[a_{i, j}\right]_{t} .
$$

This will prove Equation [2].

\subsection{A surjective map from $P_{n}$ to $S_{n}$.}

Let the function $\phi$ on the set of $n$-sequences of positive integers be defined as follows: Given an $n$ sequence of positive integers $a_{1} a_{2} \cdots a_{n}$, let the sequence $b_{1} b_{2} \cdots b_{n}$ be defined recursively by

- $b_{1}=a_{1}$, and

- For $i>1$, let $b_{i}$ be the smallest integer greater than or equal to $a_{i}$ that is not a member of $\left\{b_{1}, b_{2}, \ldots, b_{i-1}\right\}$.

Let $\phi\left(a_{1} a_{2} \cdots a_{n}\right)=b_{1} b_{2} \cdots b_{n}$.

Lemma 1. 1. If $a_{1} a_{2} \cdots a_{n} \in S_{n}$, then $\phi\left(a_{1} a_{2} \cdots a_{n}\right)=a_{1} a_{2} \cdots a_{n}$.

2. $\phi\left(a_{1} a_{2} \cdots a_{n}\right) \in S_{n}$ if and only if $a_{1} a_{2} \cdots a_{n} \in P_{n}$.

Therefore, $\phi$ is a surjective map from $P_{n}$ to $S_{n}$.

Proof. 1. If $a_{1} a_{2} \cdots a_{n} \in S_{n}$ and $\phi\left(a_{1} a_{2} \cdots a_{n}\right)=b_{1} b_{2} \cdots b_{n}$ as above, then $b_{1}=a_{1}$. Assume inductively that $a_{j}=b_{j}$ for all $j \leq i-1$. Then $a_{i} \notin\left\{b_{1}, b_{2}, \cdots, b_{i-1}\right\}=\left\{a_{1}, a_{2}, \cdots, a_{i-1}\right\}$, and $a_{i}$ is obviously the smallest such integer greater than or equal to $a_{i}$. Therefore $a_{i}=b_{i}$ for all $i$, and $\phi\left(a_{1} a_{2} \cdots a_{n}\right)=a_{1} a_{2} \cdots a_{n}$.

2. If $a_{1} a_{2} \cdots a_{n} \notin P_{n}$ and $\phi\left(a_{1} a_{2} \cdots a_{n}\right)=b_{1} b_{2} \cdots b_{n}$ as above, then there must be an integer $j$ such that $\left|\left\{i: a_{i} \leq j\right\}\right|<j$. Since $b_{i} \geq a_{i}$ for all $i, b_{i} \leq j$ only if $a_{i} \leq j$. Therefore there are strictly fewer than $k$ integers $i$ such that $b_{i} \leq j$. Therefore there are strictly fewer than $j$ integers $i$ such that $b_{i} \leq j$. Therefore $b_{1} b_{2} \cdots b_{n} \notin S_{n}$. Therefore $b_{1} b_{2} \cdots b_{n} \in S_{n}$ only if $a_{1} a_{2} \cdots a_{n} \in P_{n}$.

If $a_{1} a_{2} \cdots a_{n} \in P_{n}$ and $\phi\left(a_{1} a_{2} \cdots a_{n}\right)=b_{1} b_{2} \cdots b_{n}$, then $b_{1} b_{2} \cdots b_{n} \notin S_{n}$ if and only if, for some $R \in[n]$, there does not exist an integer $i$ such that $b_{i}=R$. Assume $R$ is minimal with this property. Therefore $[R-1] \subset\left\{b_{1}, b_{2}, \cdots, b_{n}\right\}$.

Because $a_{1} a_{2} \cdots a_{n}$ is a parking function, there must be at least $R$ integers $i$ such that $a_{i} \leq R$. Let $c_{1}<c_{2}<\cdots<c_{P}$ be the increasing rearrangement of these integers, so $P \geq R$. For each $1 \leq j \leq P, a_{c_{j}} \leq R$ and $b_{c_{j}} \neq R$. If $b_{c_{j}}>R$, then $R$ would be an integer less than $b_{c_{j}}$ that 
is greater than $a_{c_{j}}$ and not in $\left\{b_{1}, b_{2}, \cdots, b_{c_{j}-1}\right\}$. This contradicts the definition of $b_{c_{j}}$. Since $b_{c_{j}} \neq R$ by definition, $b_{c_{j}}<R$ for all $j$. Therefore $b_{c_{1}}, b_{c_{2}}, \ldots, b_{c_{P}}$ is a sequence of $P$ distinct integers strictly less than $R$ with $P \geq R$. This is impossible, and therefore $b_{1} b_{2} \cdots b_{n} \in S_{n}$.

Therefore $\phi$ is a surjective map from $P_{n}$ to $S_{n}$.

Let $\pi_{1} \pi_{2} \cdots \pi_{n}$ be a parking function of length $n$. Let the functions $g_{\pi}(i)$ be defined by $g_{\pi}(i)=$ $\min \left(\pi_{i}-\pi_{j}: i<j, \pi_{i}>\pi_{j}\right)$, with $g_{\pi}(i)=\pi_{i}$ if there is no such $\pi_{j}$. For example, if $\pi=24153$, then $\left(g_{\pi}(1), g_{\pi}(2), g_{\pi}(3), g_{\pi}(4), g_{\pi}(5)\right)=(1,1,1,2,3)$. Alternately, $g_{\pi}(i)$ is the largest integer such that $\pi_{i}-1, \pi_{i}-2, \cdots, \pi_{i}-g_{\pi}(i)+1$ all appear before $\pi_{i}$ in $\pi$.

Theorem 2. Let $\pi=\pi_{1} \pi_{2} \cdots \pi_{n}$ be a permutation in $S_{n}$.

1. $\phi^{-1}\left(\pi_{1} \pi_{2} \cdots \pi_{n}\right)=\left\{a_{1} a_{2} \cdots a_{n}: \pi_{i} \geq a_{i} \geq \pi_{i}-g_{\pi}(i)+1\right\}$.

2. $\left|\phi^{-1}\left(\pi_{1} \pi_{2} \cdots \pi_{n}\right)\right|=\prod_{i=1}^{n} g_{\pi}(i)$.

3 .

$$
(n+1)^{n-1}=\sum_{\pi \in S_{n}} \prod_{i=1}^{n} g_{\pi}(i)
$$

4.

$$
\sum_{\phi\left(a_{1} a_{2} \cdots a_{n}\right)=\pi} t^{\operatorname{area}\left(a_{1} a_{2} \cdots a_{n}\right)}=\prod_{i=1}^{n}\left[g_{\pi}(i)\right]_{t} .
$$

Proof. (sketch) Consider $\phi^{-1}$ (24153). The parking function $23153 \notin \phi^{-1}(24153)$, since $\phi$ will keep the second entry equal to 3 , as another 3 has not yet appeared. In fact, $\phi(23153)=23154$. However, $24143 \in \phi^{-1}(24153)$, since $\phi$ will change the fourth entry from 4 to 5 , since another 4 will have appeared in the second entry. Similarly, $344215 \notin \phi^{-1}(346215)$, since $\phi$ will not change the third entry from a 4 to a 6 . Instead, because the integer 5 between 4 and 6 has not yet appeared, $\phi$ will change the third entry from 4 to 5 , and $\phi(344215)=345216$. In general, $\pi_{i}$ can be replaced by an integer $k$ and still have $\phi$ change $k$ back to $\pi_{i}$ if and only if $k$, and all integers between $k$ and $\pi_{i}$, appear before $\pi_{i}$. Alternately, $\pi_{i}$ can be replaced by $k$ if and only if $\pi_{i} \geq k \geq \pi_{i}-g_{\pi}(i)+1$. The rest of the theorem follows directly or from the definition area $\left(a_{1} a_{2} \cdots a_{n}\right)=\left(\begin{array}{c}n+1 \\ 2\end{array}\right)-\sum_{i=1}^{n} a_{i}$.

\subsection{A weight-preserving bijection between $T_{n}^{*}$ and $S_{n}$.}

Recall that $T_{n}^{*}$ is the set of Tesler matrices with precisely one nonzero entry per row.

Lemma 3. A Tesler matrix in $T_{n}^{*}$ is uniquely determined by the locations of its nonzero entries. In particular, $\left|T_{n}^{*}\right|=n$ !.

Proof. (sketch) There are $n$ choices about where to put the nonzero entry in the first row, which must equal 1 . There are then $n-1$ choices about where to put the nonzero entry in the second row, which must equal 1 unless the nonzero entry in the first row was in the second column, in which case it must equal 2. Continuing in this fashion from top to bottom it is clear that the condition that every hook sum is equal to one uniquely determines the entries once their location is determined.

(This lemma was realized by the author along with Mirkó Visontai). 
We will now define a map from $S_{n}$ to $T_{n}^{*}$. Given a permutation $\pi=\pi_{1} \pi_{2} \cdots \pi_{n}$, let $h_{\pi}(i)$ be the smallest integer such that $i<h_{\pi}(i), \pi_{i}<\pi_{h_{\pi}(i)}$, and, if $\pi_{i}<k<\pi_{h_{\pi}(i)}$, then $k \in\left\{\pi_{1}, \pi_{2}, \ldots, \pi_{i-1}\right\}$. Alternately, $h_{\pi}(i)$ is the location of the first entry to the right of $\pi_{i}$ that is larger than $\pi_{i}$ but such that every integer in between it and $\pi_{i}$ appears to the left of $\pi_{i}$. (If there is no such integer let $h_{\pi}(i)=i$.) For example, if $\pi=24153$, then $\left(h_{\pi}(1), h_{\pi}(2), h_{\pi}(3), h_{\pi}(4), h_{\pi}(5)\right)=(5,4,5,4,5)$.

By construction, $i \leq h_{\pi}(i) \leq n$ for all $i$. Therefore, let $C_{\pi} \in T_{n}^{*}$ be the unique Tesler matrix with nonzero entries precisely at $\left(i, h_{\pi}(i)\right)$ for all $1 \leq i \leq n$. For example, if $\pi=24153$, then

$$
\begin{aligned}
& \begin{array}{lllll}
0 & 0 & 0 & 0 & 1
\end{array} \\
& \begin{array}{llll}
0 & 0 & 1 & 0
\end{array} \\
& C_{\pi}=\quad \begin{array}{llll}
0 & 0 & 1
\end{array} \\
& 20 \\
& 3 .
\end{aligned}
$$

We will refer to the following inductive construction of a permutation $\pi=\pi_{1} \pi_{2} \cdots \pi_{n}$ as the bumping algorithm. Given a fixed integer $1 \leq m_{1} \leq n$ and permutation $\pi^{*}=\pi_{1}^{*} \pi_{2}^{*} \cdots \pi_{n-1}^{*}$, let $\pi_{1} \pi_{2} \cdots \pi_{n}$ be defined as follows: If $m_{1}=1$, then $\pi_{1} \pi_{2} \cdots \pi_{n}=(n+1) \pi_{1}^{*} \pi_{2}^{*} \cdots \pi_{n-1}^{*}$. If $m_{1}>1$, then

- $\pi_{1}=\pi_{m_{1}-1}^{*}$.

- $\pi_{i}=\pi_{i-1}^{*}$ if $\pi_{i-1}^{*}<\pi_{m_{1}-1}^{*}$.

- $\pi_{i}=\pi_{i-1}^{*}+1$ if $\pi_{i-1}^{*} \geq \pi_{m_{1}-1}^{*}$.

For example, if $\pi^{*}=412635$ and $m_{1}=4$, then the bumping algorithm results in $\pi=2513746$.

Theorem 4. 1. If $\pi$ is constructed by the bumping algorithm from $m_{1}$ and $\pi^{*}$, then $h_{\pi}(1)=m_{1}$ and $h_{\pi}(i)=h_{\pi^{*}}(i-1)+1$ for $i>2$.

2. Therefore the map $\pi \rightarrow C_{\pi}$ is a bijection between $S_{n}$ and $T_{n}^{*}$.

Proof. (sketch) The first part follows from the definition of $h_{\pi}$ and inductively demonstrates that for every sequence $m_{1} m_{2} \cdots m_{n}$ such that $i \leq m_{i} \leq n$ for all $i$, there is at least one permutation $\pi=\pi_{1} \pi_{2} \cdots \pi_{n}$ with $h_{\pi}(i)=m_{i}$ for all $i$. This proves the second part.

Given a permutation $\pi \in S_{n}$, let $D_{\pi}$ be the increasing forest on $[n]$ constructed inductively as follows: Given a permutation $\pi^{*}=\pi_{1}^{*} \pi_{2}^{*} \cdots \pi_{n-1}^{*}$ and integer $1 \leq m_{1} \leq n$, increase the label of every node of $D_{\pi^{*}}$ by one. Add 1 as a leaf of the node now labelled $m_{1}$. If $m_{1}=1$, leave 1 as an isolated node. Let $D_{\pi}$ be the resulting increasing forest, where $\pi$ is constructed by the bumping algorithm from $\pi^{*}$ and $m_{1}$. (Increasing forests are well-known to be equinumerous with permutations, see [Sta97].)

Theorem 5. For each $i \in[n]$, the parent of the node labelled $i$ in the increasing forest $D_{\pi}$ is the node labelled $h_{\pi}(i)$.

Proof. The parent of the node labelled 1 is the node labelled $m_{1}$. For $i>1$, assume inductively that the parent of the node labelled $i-1$ in $D_{\pi^{*}}$ is the node labelled $h_{\pi^{*}}(i-1)$. Then the parent of the node labelled $i$ in $D_{\pi}$ is the node labelled $h_{\pi^{*}}(i-1)+1=h_{\pi}(i)$. 
Theorem 6. 1. Given $\pi \in S_{n}$, the Tesler matrix $C_{\pi} \in T_{n}^{*}$ has the entry $g_{\pi}(i)$ at $\left(i, h_{\pi}(i)\right)$. Equivalently, if an upper-triangular matrix has only the nonzero entries $g_{\pi}(i)$ at $\left(i, h_{\pi}(i)\right)$, then it is a Tesler matrix.

2.

$$
\prod_{C_{\pi}=\left[a_{i, j}\right], a_{i, j}>0}\left[a_{i, j}\right]_{t}=\prod_{i=1}^{n}\left[g_{\pi}(i)\right]_{t}=\sum_{\phi\left(a_{1} a_{2} \cdots a_{n}\right)=\pi} t^{\text {area }\left(a_{1} a_{2} \cdots a_{n}\right)}
$$

Proof. (sketch) From the definitions of $g_{\pi}$ and $h_{\pi}$ and taking $h_{\pi}^{0}(j)=j$, it can be seen that

$$
g_{\pi}(i)=\left|\left\{j: h_{\pi}^{r}(j)=i\right\}\right| .
$$

Alternately, $g_{\pi}(i)$ is the number of integers eventually sent to $i$ by $h_{\pi}$. Using Theorem (5), $h_{\pi}$ can be seen as a function that sends an integer labelling a node in the increasing forest $D_{\pi}$ to the integer labelling the parent of that node. Therefore $g_{\pi}(i)$ is the number of descendents in $D_{\pi}$ of the node labelled $i$, including the node itself.

If an upper-triangular matrix has only the nonzero entries $g_{\pi}(i)$ at $\left(i, h_{\pi}(i)\right)$, then the $i$-th hook sum is $g_{\pi}(i)-\sum_{j: h_{\pi}(j)=i} g_{\pi}(j)$. This is equal to the number of descendents in $D_{\pi}$ of the node labelled $i$ (including itself) minus the number of descendents of the nodes with $i$ as a parent (including themselves) This must equal 1 . Therefore the upper-triangular matrix with only the nonzero entries $g_{\pi}(i)$ at $\left(i, h_{\pi}(i)\right)$ is the unique Tesler matrix $C_{\pi} \in T_{n}^{*}$.

This proves Equation [2].

\section{The $t=0$ special case}

Note added in proof: The version of this extended abstract originally submitted described our efforts to find a proof of Equation (3). We have since completed a proof of Equation (3), which we will give the broad outlines of below. A more complete description of both proofs may likely be in a forthcoming article.

Recall that, if $a_{1} a_{2} \cdots a_{n}$ is a parking function of length $n$, then area $\left(a_{1} a_{2} \cdots a_{n}\right)=\left(\begin{array}{c}n+1 \\ 2\end{array}\right)-\sum_{i=1}^{n} a_{i}$.

Lemma 7. A parking function $a_{1} a_{2} \cdots a_{n} \in P_{n}$ has area equal to zero if and only if it is a permutation in $S_{n}$.

Therefore, when Equation (1) is evaluated at $t=0$, the following equation results:

$$
\sum_{\pi_{1} \pi_{2} \cdots \pi_{n} \in S_{n}} q^{\operatorname{dinv}\left(\pi_{1} \pi_{2} \cdots \pi_{n}\right)}=\sum_{A=\left[a_{i, j}\right] \in T_{n}}(q-1)^{\operatorname{extra}(A)} q^{\sum_{a_{i, j}>0}\left(a_{i, j}-1\right)} .
$$

The dinv statistic is complicated to define on general parking functions (see [Hag08]). However, it is easier to define on permutations: For $\pi_{1} \pi_{2} \cdots \pi_{n} \in S_{n}$, let

$$
\operatorname{dinv}\left(\pi_{1} \pi_{2} \cdots \pi_{n}\right)=\left|\left\{(i, j): i<j, \pi_{i}<\pi_{j}\right\}\right| .
$$


Alternately, dinv gives the number of pairs of entries of $\pi_{1} \pi_{2} \cdots \pi_{n}$ that are not inversions. It is well known that dinv is a Mahonian statistic when applied to permutations, so

$$
\sum_{\pi_{1} \pi_{2} \cdots \pi_{n} \in S_{n}} q^{\operatorname{dinv}\left(\pi_{1} \pi_{2} \cdots \pi_{n}\right)}=\prod_{i=1}^{n}[i]_{q} .
$$

We will sketch a proof of Equation (3). In particular, we will partition the set of $n \times n$ Tesler matrices $T_{n}$ into $n$ ! disjoint subsets $T_{\pi}$ indexed by the permutations $\pi \in S_{n}$. These subsets $T_{\pi}$ are such that, for each $\pi \in S_{n}$,

$$
\sum_{A=\left[a_{i, j}\right] \in T_{\pi}}(q-1)^{\operatorname{extra}(A)} q^{\sum_{a_{i, j}>0}\left(a_{i, j}-1\right)}=q^{\operatorname{dinv}(\pi)} .
$$

This will prove Equaiton (3).

\subsection{Decoding Tesler Matrices}

Given a Tesler matrix $A=\left[a_{i, j}\right] \in T_{n}$, let $A^{*}$ be the multiset of ordered pairs and integers such that $(i, j) \in A^{*}$ with multiplicity $k$ if and only if $a_{i, j}=k$ for $i<j$ and $i \in A^{*}$ with multiplicity $k$ if and only if $a_{i, i}=k$. For example, if

$$
\begin{aligned}
& \begin{array}{lllll}
0 & 1 & 0 & 0 & 0
\end{array} \\
& \begin{array}{llll}
0 & 2 & 0 & 0
\end{array} \\
& A=\quad \begin{array}{lll}
1 & 1 & 1,
\end{array} \\
& 11
\end{aligned}
$$

then $A^{*}=\{(1,2),(2,3),(2,3), 3,(3,4),(3,5), 4,(4,5), 5,5,5\}$. From each such $A^{*}$, we can construct a list of $n$ vertically-arranged sets, each written in increasing order, such that:

- $a$ is immediately before $b$ in a set if and only if $(a, b) \in A^{*}$.

- The last entries in each set are the single integers in $A^{*}$, weakly decreasing from the top set to the bottom set.

In particular, we want to construct such a list of $n$ vertically-arranged sets, each written in increasing order, such that:

- The last entry in each set must be greater than or equal to any entry in a set below.

- The first entry in each set does not appear in any set below, and is the only entry in that set where this is the case.

- If $a$ is immediately before $b$ in a set and $c$ is immediately before $b$ in a set above, then $c \geq b$.

For example, one list of sets resulting from $\{(1,2),(2,3),(2,3), 3,(3,4),(3,5), 4,(4,5), 5,5,5\}$ satisfying the above rules is 


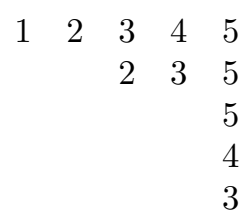

We will omit the proof of the following theorem stating that this construction is bijective.

Theorem 8. 1. There is precisely one list of sets satisfying the above rules for each matrix $A \in T_{n}$.

2. If a list of sets satisfies the above rules, then the first entry of each set, read from top to bottom, gives a permutation $\pi$.

For a fixed $\pi \in S_{n}$, let $T_{\pi}$ be the set of Tesler matrices $A \in T_{n}$ such that the unique list of sets satisfying the above rules gives the permutation $\pi$. For example, $T_{31254}$ consists of the following Tesler matrices (each paired with the corresponding list of sets)

\begin{tabular}{|c|c|c|c|c|c|c|c|c|c|c|c|c|c|c|c|c|c|}
\hline \multirow{5}{*}{1} & 3 & & & ) & 0 & 0 & 0 & & & & 3 & 4 & 5 & 0 & 1 & 0 & 0 \\
\hline & 2 & 5 & & ( & 0 & 0 & 2 & & & & 1 & 2 & 5 & & 0 & 0 & 2 \\
\hline & & 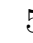 & & & 0 & 0 & 1 & & & & & 2 & 5 & & ( & 1 & 0 \\
\hline & & 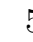 & & & & 1 & 0 & & & & & & 5 & & & 1 & 1 \\
\hline & & & & & & & 4 & & & & & & 4 & & & & 4 \\
\hline \multirow{10}{*}{1} & 3 & 4 & 5 & 0 & 1 & 0 & 0 & & & & 3 & 4 & 5 & 0 & 0 & 0 & 1 \\
\hline & 2 & 4 & 5 & & 0 & 0 & 1 & & & & 1 & 4 & 5 & & 0 & 0 & 0 \\
\hline & & 2 & 5 & & & 0 & 1 & & & & & 2 & 5 & & & 0 & 1 \\
\hline & & & 5 & & & & 1 & & & & & & 5 & & & & 1 \\
\hline & & & 4 & & & & & & & & & & 4 & & & & \\
\hline & & & & & & 3 & 4 & 5 & 0 & 1 & 0 & 0 & 0 & & & & \\
\hline & & & & & 1 & 2 & 4 & 5 & & 0 & 0 & 2 & 0 & & & & \\
\hline & & & & & & 2 & 4 & 5 & & & 0 & 1 & 0. & & & & \\
\hline & & & & & & & & 5 & & & & 1 & 3 & & & & \\
\hline & & & & & & & & 4 & & & & & 4 & & & & \\
\hline
\end{tabular}

Note that the Tesler matrix $C_{31254}$ is in $T_{31254}$, and that the sum of $(q-1)^{\operatorname{extra}(A)} q^{\sum_{a_{i, j}>0}\left(a_{i, j}-1\right)}$ over $T_{31254}$ is equal to $q^{7}=q^{\operatorname{dinv}(31254)}$. Finally, note that the term of $q^{7}$ comes from the term of the generating function corresponding to the last Tesler matrix. In general, for $\pi=\pi_{1} \pi_{2} \cdots \pi_{n} \in S_{n}, T_{\pi}$ will always contain precisely one Tesler matrix such that the first set in the corresponding list of sets consists of $\pi_{1}$ followed by everything after and larger than $\pi_{1}$, the second set consists of $\pi_{2}$ followed by everything after and larger than $\pi_{2}$, and so on. This Tesler matrix will always give a term of $q^{\operatorname{dinv}(\pi)}$. It is possible to define an involution that cancels out the other terms, but we will not describe the involution in this extended abstract.

Theorem 9. The following equation holds due to an involution:

$$
\sum_{A=\left[a_{i, j}\right] \in T_{\pi}}(q-1)^{\operatorname{extra}(A)} q^{\sum_{a_{i, j}>0}\left(a_{i, j}-1\right)}=q^{\operatorname{dinv}(\pi)} .
$$




\subsection{A non-Mahonian statistic on $S_{n}$}

Having sketched a combinatorial proof of Equation (3), we will now discuss how it relates to the combinatorial proof presented earlier of Equation (2).

We can define a statistic akin to dinv by replacing the $t$-analogues in Equation (2) with $q, t$-analogues. For $a_{1} a_{2} \cdots a_{n} \in P_{n}$ with $\phi\left(a_{1} a_{2} \cdots a_{n}\right)=\pi_{1} \pi_{2} \cdots \pi_{n}$, let $p \operatorname{dinv}\left(a_{1} a_{2} \cdots a_{n}\right)$ be defined by

$$
\operatorname{pdinv}\left(a_{1} a_{2} \cdots a_{n}\right)=\sum_{i=1}^{n}\left(a_{i}-\pi_{i}+g_{\pi}(i)+1\right) .
$$

Alternately, $\operatorname{pdinv}\left(a_{1} a_{2} \cdots a_{n}\right)$ is the sum of how much there is left that can be subtracted from each entry and still leave a parking function in $\phi^{-1}\left(\pi_{1} \pi_{2} \cdots \pi_{n}\right)$.

Lemma 10. 1. pdinv satisfies the following generating function identity:

$$
\sum_{a_{1} a_{2} \cdots a_{n} \in P_{n}} q^{\text {pdinv }\left(a_{1} a_{2} \cdots a_{n}\right)} t^{\text {area }\left(a_{1} a_{2} \cdots a_{n}\right)}=\sum_{A=\left[a_{i, j}\right] \in T_{n}^{*}} \prod_{a_{i, j}>0}\left[a_{i, j}\right]_{q, t} .
$$

2. pdinv is equidistributed with area over $P_{n}$ and is therefore equidistributed with dinv over $P_{n}$.

Proof. The first part of the lemma is clear from the alternate description of pdinv. The equidistribution of area and pdinv follows from the fact that each $\left[a_{i, j}\right]_{q, t}$ is symmetric in $q$ and $t$. The equidistribution dinv and pdinv then follows from the equidistribution of area and dinv.

However, (pdinv, area) is not joint-equidistributed with (dinv, area) over $P_{n}$. If it were, then there would be no need for the terms of Equation (1) corresponding to Tesler matrices with more than one entry per row. For example, the sum of $q^{\text {pdinv }\left(a_{1} a_{2} a_{3}\right)}$ over $S_{3}$, the set of parking functions of length 3 with zero area, is equal to $1+3 q+q^{2}+q^{3}$, while the sum of $q^{\operatorname{dinv}\left(a_{1} a_{2} a_{3}\right)}$ over $S_{3}$ is equal to $1+2 q+2 q^{2}+q^{3}$. (dinv being Mahonian over $S_{3}$.)

We can therefore think of the Tesler matrices with more than one entry per row as somehow de-coupling and re-coupling pdinv and area in some parking functions to obtain (conjecturally) the (dinv, area) joint distribution.

As with dinv, pdinv is difficult to interpret combinatorially as a statistic on $P_{n}$ but easier to interpret combinatorially as a statistic on $S_{n}$. In particular, pdinv enumerates a subset of non-inversion pairs.

Theorem 11. For $\pi_{1} \pi_{2} \cdots \pi_{n} \in S_{n}$, pdinv $\left(\pi_{1} \pi_{2} \cdots \pi_{n}\right)$ is the number of pairs $(i, j)$ such that $i<j$, $\pi_{i}<\pi_{j}$ and, for all $i<j<k, \pi_{k} \notin\left[\pi_{i}, \pi_{j}\right]$. Alternately, pdinv $\left(\pi_{1} \pi_{2} \cdots \pi_{n}\right)$ is the number of pairs $(i, j)$ such that $\pi_{j}$ forms a non-inversion with $\pi_{i}$ and there is no integer after it in between $\pi_{i}$ and $\pi_{j}$ in magnitude.

Proof. (sketch) By definition, pdinv $\left(\pi_{1} \pi_{2} \cdots \pi_{n}\right)=-n+\sum_{i=1}^{n} g_{\pi}(i)$. Recall that $g_{\pi}(i)$ is the largest integer such that $\pi_{i}-1, \pi_{i}-2, \cdots, \pi_{i}-g_{\pi}(i)+1$ all appear before $\pi_{i}$ in $\pi . \pi_{i}$ will form a non-inversion with each of these integers, and for each integer there can be no integer after $\pi_{i}$ in between it and $\pi_{i}$ in magnitude. Since there are $g_{\pi}(i)-1$ such integers, there will be $-n+\sum_{i=1}^{n} g_{\pi}(i)=p \operatorname{din} v\left(\pi_{1} \pi_{2} \cdots \pi_{n}\right)$ such pairs in all.

Looking only at the $t=0$ special case, we can therefore think of the effect of the matrices in $T_{\pi}$ as adding these "missing" anti-inversions back to $\operatorname{pdinv}(\pi)$ to result in $\operatorname{dinv}(\pi)$. Each of these missing anti-inversions comes from the pdinv of another parking function, and so on. 


\section{Further Directions and Conjectures}

Note added in proof: This section has been updated to account for our current work and conjectures.

The proof of the $t=0$ special case, Equation (3), shows that the Tesler matrices can be divided into disjoint subsets $T_{\pi}$ indexed by the permutations $\pi \in S_{n}$ such that the Haglund generating function is $q$-positive at $t=0$ when summed over each subset. The full Haglund generating function is not $q, t$ positive when summed over each $T_{\pi}$. However, we have verified for $n \leq 7$ that the Haglund generating function is $q, t$-positive when summed over the union of $T_{\pi}$ over naturally-defined subsets of $S_{n}$. This suggests that the parking functions conjecture might be refinable. Unfortunately, the resulting positive generating functions do not match the generating function of dinv and area over the corresponding parking functions. This suggests that some alternate statistic $f$, such that $(f$, area $)$ is joint-equidistributed with (dinv, area), might better illuminate the combinatorics of the parking functions conjecture.

Also, we are unfamiliar with the work surrounding the Kreveras theorem, which states that parking functions with area $k$ are equinumerous with spanning forests with $k$ inversions, but it apparently lacks a "nice" bijective proof, and it is possible our proof of Equation (2) could yield one through our use of spanning increasing forests. See Pak [Pak] and Kreveras [Kre80].

\section{Acknowledgements}

The author would like to thank James Haglund for sharing this problem with him and Mirkó Visontai for his discussion and encouragement.

\section{References}

[GH96] A. M. Garsia and M. Haiman. A remarkable $q, t$-Catalan sequence and $q$-Lagrange inversion. $J$. Algebraic Combin., 5(3):191-244, 1996.

[Hag] James Haglund. A polynomial identity for the hilbert series of the space of diagonal harmonics. Advances in Mathematics. To Appear.

[Hag08] James Haglund. The q,t-Catalan numbers and the space of diagonal harmonics, volume 41 of University Lecture Series. American Mathematical Society, Providence, RI, 2008. With an appendix on the combinatorics of Macdonald polynomials.

[Kre80] G. Kreveras. Une famille de polynomes ayant plusieurs proprieties enumeratives. Period. Math. Hung., 11:309-320, 1980.

[Pak] Igor Pak. Tree bijections. http://www.math.ucla.edu/ pak/lectures/ bij-trees-talk1.pdf.

[Sta97] Richard P. Stanley. Enumerative combinatorics. Vol. 1, volume 49 of Cambridge Studies in Advanced Mathematics. Cambridge University Press, Cambridge, 1997. With a foreword by Gian-Carlo Rota, Corrected reprint of the 1986 original. 